\title{
APROVEITAMENTO DE CASCA DE MANGA TOMMY ATKINS (Mangifera indica Linn) PARA OBTENÇAO DE EXTRATOS RICOS EM COMPOSTOS FENÓLICOS ATRAVÉS DE DIFERENTES MÉTODOS DE EXTRAÇÃO
}

\author{
M. E.A.O. de $\mathrm{SOUZA}^{1,2}$, N. MEZZOMO ${ }^{1}$, L.C. de AZEVEDO ${ }^{2}$ e S.R.S. FERREIRA ${ }^{1}$, \\ ${ }^{1}$ Universidade Federal de Santa Catarina, Departamento de Engenharia Química e Engenharia de \\ Alimentos \\ ${ }^{2}$ Instituto Federal de Educação Ciência e Tecnologia do Sertão Pernambucano, Departamento de \\ Tecnologia de Alimentos \\ E-mail para contato: meaos1@yahoo.com.br
}

\begin{abstract}
RESUMO - A industrialização da manga Tommy Atkins (Mangifera indica Linn) gera como resíduo a casca da fruta que contém em sua composição compostos fenólicos. $\mathrm{O}$ objetivo do presente trabalho é estudar o aproveitamento da casca para a obtenção de extratos mediante o emprego das técnicas de extração no sistema Soxhlet, maceração e ultrassom, utilizando solventes distintos, e o processo de extração supercrítica (ESC) em diferentes condições de pressão (100-300bar) e de temperatura $\left(40-60^{\circ} \mathrm{C}\right)$. Os extratos foram avaliados em termos de rendimento e teor de fenólicos totais (TFT). Na extração a baixa pressão, os maiores valores de rendimento foram obtidos no sistema Soxhlet utilizando o solvente etanol $(37,1 \pm 0,7 \%)$, e o maior TFT foi obtido na maceração com etanol $(63 \pm 2 \mathrm{mgEAG} / \mathrm{g})$. Na ESC o rendimento foi de até $3,8 \pm 0,7 \%$ (300bar $\left./ 50^{\circ} \mathrm{C}\right)$ utilizando $\mathrm{o}_{2}$ puro, sendo que o extrato com maior teor de fenólicos na alta pressão $(23 \pm 1 \mathrm{mgEAG} / \mathrm{g})$ foi na condição de $300 \mathrm{bar} / 60^{\circ} \mathrm{C}$.
\end{abstract}

\section{INTRODUÇÃO}

Nas áreas irrigadas do semiárido nordestino existe um grande potencial de produção de manga devido às condições climáticas favoráveis da região. A manga é um fruto muito atrativo, especialmente pelo seu aroma, sabor adocicado e sua aparência. Ela é consumida de forma in natura ou industrializada na forma de sucos ou polpa, sendo utilizada na produção de vários tipos de alimentos tais como: doce, geléia, sorvete, licor, entre outros. Na sua industrialização, a fruta gera um enorme volume de resíduos que, quando não se tem um destino adequado para este material, pode provocar sérios danos ao meio ambiente (COELHO, 2012). A casca da manga é um importante subproduto da indústria de transformação de manga e constitui entre $15-20 \%$ do peso total da fruta (KIM et al., 2012).

Os métodos convencionais de extração a exemplo do sistema Soxhlet, maceração e ultrassom, que são utilizados com solventes orgânicos e aplicados à baixa pressão, são bastante empregados na obtenção de extratos e óleos essenciais de várias matérias-primas. Nas extrações com solventes 


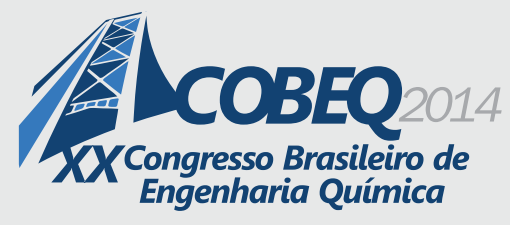

19 a 22 de outubro de 2014

Florianópolis/SC

orgânicos são utilizados de solventes de diferentes polaridades, como etanol, hexano e acetato de etila, e são comumente aplicadas nas indústrias química, farmacêutica e de alimentos para a produção de extratos. Porém, estas técnicas envolvem altas temperaturas de processo, podendo ocasionar alterações químicas e danificar os compostos termossensíveis, assim como a perda de qualidade do óleo essencial (BE-JEN; YEN-HUI; ZER-RAN, 2004; POURMORTAZAVI; HAJIMIRSADEGUI, 2007). A extração com fluido supercrítico tem sido considerada uma boa opção para a extração e fracionamento de produtos naturais. A extração supercrítica (ESC) emprega gases pressurizados como solventes e se diferencia das outras técnicas convencionais pela produção de extratos isentos de solvente e sem degradação térmica, além de permitir o fácil controle da seletividade do processo. Estes fatores são considerados aspectos importantes para as indústrias que utilizam os produtos naturais como matéria prima (KHAJEH et al. 2004).

Diversas técnicas vêm sendo utilizadas para determinar a atividade antioxidante de forma a permitir uma rápida seleção de substâncias e/ou misturas potencialmente interessante. Uma forma indireta de estimar a atividade antioxidante é através do Teor de Fenólicos Totais (TFT) que utiliza o método espectrofotométrico de Folin-Ciocalteau.

Diante do exposto, o objetivo geral deste trabalho foi estudar o aproveitamento da casca de manga da variedade Tommy Atkins (resíduo do processamento da fruta) na obtenção de extratos de alto valor agregado, avaliando a eficiência de diferentes técnicas de extração e a qualidade dos extratos obtidos (em termos de rendimento do processo e teor de fenólicos dos extratos).

\section{MATERIAIS E MÉTODOS}

Serão apresentados os materiais e métodos utilizados nos experimentos de extração do óleo da casca de manga através das técnicas convencionais de extração (Soxhlet, maceração e ultrassom) e da extração a alta pressão (supercrítica), assim como a determinação do teor de fenólicos totais (TFT) dos extratos obtidos da casca de manga. Todos os experimentos de caracterização da matéria-prima, extrações convencionais, extração com $\mathrm{CO}_{2}$ supercrítico (ESC) e determinação do rendimento e teor de fenólicos foram realizadas no Laboratório de Termodinâmica e Extração Supercrítica (LATESC) do Departamento de Engenharia Química e Engenharia de Alimentos da Universidade Federal de Santa Catarina (UFSC) em Florianópolis-SC.

A manga Tommy Atkins (Mangifera indica Linn) foi fornecida em diferentes lotes por alguns produtores de fruta da região do submédio do Vale do São Francisco no município de Petrolina-PE, localizada a $9^{\circ} 34^{\prime}$ de latitude sul, $40^{\circ} 26^{\prime}$ de longitude oeste e altitude de $375 \mathrm{~m}$ e o clima é do tipo semiárido (FONSECA et al., 2005). As frutas foram coletadas em dias de produção diferentes e misturadas com a finalidade de obter uma amostragem representativa e que abrangesse a composição média do resíduo de processo da manga, uma vez que ocorrem variações na composição dos frutos colhidos em função da época do ano e da região de plantio. As frutas foram lavadas, enxugadas e depois retiradas as suas cascas. A separação da casca e polpa da manga foi realizada manualmente, com o auxílio de faca inox de cozinha. As amostras das cascas de manga foram secas de duas formas: a) secagem natural ao ar livre durante 7 dias na temperatura ambiente, à sombra disposto em cima de papel para a absorção da umidade e protegido contra o ataque de insetos ou contaminantes 


\section{9 a 22 de outubro de 2014 \\ Florianópolis/SC}

ambientais); e b) secagem artificial por estufa com circulação forçada de ar (Desidratador Pardal PE 30, 220V, 900W, Petrópolis-RJ) a Temperatura de $40^{\circ} \mathrm{C}$ durante 24 horas. Após a secagem, as cascas foram trituradas em um moinho de facas (De Leo, tipo Willey EDB 5, 1/3 HP, Bento Gonçalves/RS) e acondicionada em embalagens plásticas identificadas, e mantida congelada, em freezer doméstico à $-18{ }^{\circ} \mathrm{C}$ até a realização dos experimentos. Na extração a baixa pressão foi utilizada amostras das secagens naturais e por estufa, já na alta pressão foram utilizadas amostra da secagem por estufa.

Os processos de extração a baixa pressão (Soxhlet, maceração e ultrassom) de óleo da casca de manga foram realizados através da extração com solventes orgânicos de diferentes polaridades (etanol, hexano e acetato de etila), enquanto na ESC o solvente utilizado foi o dióxido de carbono. Em todas as técnicas de extrações empregadas foram determinados o rendimento do processo de extração, teor de fenólicos dos extratos, a fim de avaliar e comparar as metodologias empregadas. As técnicas de extração foram realizadas em duplicata.

A extração por sistema Soxhlet foi realizada de acordo com o descrito pelo Instituto Adolfo Lutz (BRASIL, 2005) e neste processo foram utilizados $150 \mathrm{~mL}$ do solvente selecionado, o qual foi acondicionado no balão volumétrico e aquecido através de uma manta térmica na temperatura de ebulição do solvente utilizado. De acordo com o método 920.39C da AOAC (2005) para cada extração foi utilizada $5 \mathrm{~g}$ de amostra da casca de manga moída que foram medidas em balança analítica, com precisão ao décimo de miligrama envolvidos em um cartucho de papel filtro o qual foi inserido no extrator. $\mathrm{O}$ tempo total do processo foi de $6 \mathrm{~h}$. A extração por maceração foi efetuado de acordo com Sachindra et al. (2006) empregando $25 \mathrm{~g}$ de matéria-prima e adicionados $100 \mathrm{~mL}$ de solvente. A mistura da matéria-prima com o solvente foi mantida protegida ao abrigo da luz por um período de 5 dias na temperatura ambiente e com agitação manual uma vez ao dia. A separação da amostra e extrato foi realizada através da filtração com auxílio de bomba de vácuo em funil de Büchner com papel-filtro, sendo o filtrado recolhido em um Kitasato. A extração ultrassônica foi adaptada de $\mathrm{Gu}$ et al. (2008) . O método consistiu de adicionar 5 g de matéria-prima pré-tratada a 150 $\mathrm{mL}$ de solvente em um balão de $250 \mathrm{~mL}$ pelo tempo de extração de $60 \mathrm{~min}$. Após a extração, solução de extrato foi separada da matriz à temperatura ambiente através da filtração.

Os extratos obtidos da matéria prima através do sistema Soxhlet, maceração e ultrassom foram evaporados em rota-evaporador com rotação de $60 \mathrm{rpm}$, sob controle de vácuo com valor médio de $650 \mathrm{mmHg}$ e arrefecimento. As temperaturas de evaporação foram ajustadas para valores inferiores aos pontos de ebulição dos solventes utilizados, para evitar alterações do extrato devido à aplicação de calor. As massas de extrato seco foram medidas em balança analítica e o rendimento calculado em termos de massa de casca de manga utilizada, sendo os resultados expressos em base seca (b.s.) como media \pm desvio padrão. Os extratos obtidos foram acondicionados em frascos âmbar e armazenados no freezer doméstico a $-18^{\circ} \mathrm{C}$, até a realização das análises.

O teor de compostos fenólicos totais (TFT) foi determinado para todas as amostras de extrato de acordo com o método de Folin-Ciocalteau (ROSSI; SINGLETON, 1965; PESCHEL et al., 2006). Resumidamente, a mistura de reação foi composta por $0,1 \mathrm{ml}$ de extrato $(1667 \mathrm{mg} / \mathrm{L}), 7,9 \mathrm{~mL}$ de água destilada, 0,5 $\mathrm{mL}$ de reagente de Folin-Ciocalteau (uma mistura de fosfomolibdato e fosfotungstato) e 1,5 mL de carbonato de sódio a $20 \%$, colocados em frascos opacos. Os frascos 
foram agitados, mantida durante $2 \mathrm{~h}$, e a absorvância foi medida a $765 \mathrm{~nm}$. O TFT foi calculado de acordo com uma curva padrão, preparado anteriormente com ácido gálico. A análise foi realizada em triplicata e o resultado expresso em $\mathrm{mgEAG} / \mathrm{g}$ de extrato, como média \pm desvio padrão.

O experimento de ESC consiste na utilização na matriz sólida do gás pressurizado dióxido de carbono $\left(\mathrm{CO}_{2}\right)$ com $99,9 \%$ de pureza (White Martins) para a solubilização da mistura soluto/solvente. Os experimentos foram realizados em uma unidade que opera a pressão máxima de 300 bar e com vazões de solvente de $1,7 \mathrm{~g} / \mathrm{min}$ a $36,7 \mathrm{~g} / \mathrm{min}$. As extrações foram realizadas em diferentes condições de pressão $\left(100,200\right.$ e 300 bar) e de temperatura $\left(40,50\right.$ e $\left.60^{\circ} \mathrm{C}\right)$, com vazão de $8,3 \mathrm{~g} / \mathrm{min}$ de $\mathrm{CO}_{2}$ e $15 \mathrm{~g}$ de matéria-prima nas quais o maior rendimento global e TFT pudessem ser obtidos levando em consideração os limites de operação do equipamento utilizado.

\section{RESULTADOS E DISCUSSÃO}

A Tabela 1 apresenta os resultados de rendimento de extração dos diferentes sistemas empregados, em função da umidade de matéria-prima utilizada. De acordo com os resultados da Tabela 1, os melhores rendimentos para os dois tipos de secagem da matéria-prima foram obtidos na extração Soxhlet utilizando etanol como solvente, alcançando valores de até $37,1 \pm 0,7 \%$, enquanto as extrações por maceração e ultrassom promoveram os menores rendimentos.

Tabela 1 - Rendimento global $\left(\mathrm{X}_{0}\right)$ obtidos nas extrações convencionais de casca de manga

\begin{tabular}{|c|c|c|c|c|}
\hline \multicolumn{4}{|c|}{ Condições de Extrações convencionais } & \multirow[b]{2}{*}{$\begin{array}{l}\text { Rendimento } \\
\mathrm{X}_{0}(\%)^{(3)}\end{array}$} \\
\hline Extração & $\begin{array}{c}\text { Secagem da } \\
\text { matéria-prima }^{(1)}\end{array}$ & Solventes & $\operatorname{IPS}^{(2)}$ & \\
\hline \multirow{6}{*}{ Soxhlet } & \multirow{3}{*}{ Natural } & Etanol & 5,2 & $37,1^{\mathrm{a}} \pm 0,7$ \\
\hline & & Hexano & 0,0 & $2,5^{\mathrm{d}} \pm 0,4$ \\
\hline & & Ac. Etila & 4,4 & $4,5^{\mathrm{d}} \pm 0,2$ \\
\hline & \multirow{3}{*}{ Estufa } & Etanol & 5,2 & $36,3^{\mathrm{a}} \pm 0,7$ \\
\hline & & Hexano & 0,0 & $2,4^{\mathrm{de}} \pm 0,5$ \\
\hline & & Ac. Etila & 4,4 & $4,3^{\mathrm{d}} \pm 0,2$ \\
\hline \multirow{6}{*}{ Maceração } & \multirow{3}{*}{ Natural } & Etanol & 5,2 & $18,7^{b} \pm 1,8$ \\
\hline & & Hexano & 0,0 & $2,3^{\mathrm{d}} \pm 0,4$ \\
\hline & & Ac. Etila & 4,4 & $4,5^{\mathrm{d}} \pm 0,3$ \\
\hline & \multirow{3}{*}{ Estufa } & Etanol & 5,2 & $19^{\mathrm{b}} \pm 2$ \\
\hline & & Hexano & 0,0 & $2,3^{\mathrm{de}} \pm 0,5$ \\
\hline & & Ac. Etila & 4,4 & $4,3^{\mathrm{d}} \pm 0,1$ \\
\hline \multirow{6}{*}{ Ultrassom } & \multirow{3}{*}{ Natural } & Etanol & 5,2 & $11,6^{c} \pm 0,6$ \\
\hline & & Hexano & 0,0 & $1,5^{\mathrm{d}} \pm 0,5$ \\
\hline & & Ac. Etila & 4,4 & $2,7^{\mathrm{d}} \pm 0,5$ \\
\hline & \multirow{3}{*}{ Estufa } & Etanol & 5,2 & $14,3^{\mathrm{c}} \pm 0,5$ \\
\hline & & Hexano & 0,0 & $1,5^{\mathrm{de}} \pm 0,1$ \\
\hline & & Ac. Etila & 4,4 & $1,88^{\mathrm{de}} \pm 0,02$ \\
\hline
\end{tabular}


(1) Secagem natural = secagem a sobra na temperatura ambiente, Secagem em estufa $=$ secagem por ar quente através de estufas com circulação forçada de ar, ${ }^{(2)}$ IPS = Índice de polaridade de cada solvente (BYERS, 2013). ${ }^{(2)}$ Letras iguais na mesma coluna indicam que não há diferença significativa $(\mathrm{p}<0,05)$.

Verificando os rendimentos obtidos na extração supercrítica, em relação aos resultados obtidos nas extrações convencionais, observa-se que os extratos obtidos a baixa pressão, empregando o solvente etanol, tiveram rendimentos superiores aos alcançados pela alta pressão com $\mathrm{CO}_{2}$ puro.

Os rendimentos globais $\left(\mathrm{X}_{0}\right)$ da extração supercrítica, obtidos nas diferentes condições de temperatura e pressão utilizando $\mathrm{CO}_{2}$ puro, estão apresentado na Tabela 2. De acordo com os resultados obtidos, o maior $\mathrm{X}_{0}$ foi obtido na condição de 300 bar e $50{ }^{\circ} \mathrm{C}$.

Tabela 2 - Rendimento global $\left(\mathrm{X}_{0}\right)$ obtidos nas extrações supercríticas de casca de manga

\begin{tabular}{|c|c|c|c|}
\hline \multicolumn{3}{|c|}{ Condições de Extrações Supercríticas } & \multirow{2}{*}{$\begin{array}{l}\text { Rendimento } \\
\mathrm{X}_{0}(\%)^{(2)}\end{array}$} \\
\hline $\mathrm{P}(\mathrm{bar})$ & $\mathrm{T}\left({ }^{\circ} \mathrm{C}\right)$ & $\rho_{\mathrm{CO} 2}\left(\mathrm{~g} / \mathrm{cm}^{3}\right)^{(1)}$ & \\
\hline \multirow{3}{*}{100} & 40 & 0,629 & $0,8^{\mathrm{bc}} \pm 0,1$ \\
\hline & 50 & 0,385 & $0,72^{\mathrm{bc}} \pm 0,01$ \\
\hline & 60 & 0,295 & $0,50^{b c} \pm 0,02$ \\
\hline \multirow{3}{*}{200} & 40 & 0,840 & $2,4^{\mathrm{ab}} \pm 0,1$ \\
\hline & 50 & 0,785 & $1,42^{b c} \pm 0,08$ \\
\hline & 60 & 0,724 & $1,40^{\mathrm{bc}} \pm 0,02$ \\
\hline \multirow{3}{*}{300} & 40 & 0,911 & $2,7^{\mathrm{ab}} \pm 0,8$ \\
\hline & 50 & 0,871 & $3,8^{\mathrm{a}} \pm 0,7$ \\
\hline & 60 & 0,830 & $2,55^{\mathrm{ab}} \pm 0,02$ \\
\hline
\end{tabular}

${ }^{(1)}$ Densidade do $\mathrm{CO}_{2}$ segundo Angus, Armstrong e De Reuck (1976). ${ }^{(2)}$ Letras iguais na mesma coluna indicam que não há diferença significativa $(\mathrm{p}<0,05)$.

De acordo com a Tabela 3 que apresenta os resultados de TFT das amostras das extrações a baixa pressão, o melhor resultado do teor de fenólicos foi encontrado na maceração da amostra da secagem natural utilizando o solvente etanol (63 $\pm 2 \mathrm{mg}$ EAG/g).

Os resultados nas extrações convencionais podem ser justificados pela obtenção de compostos mais polares, não solúveis no dióxido de carbono que é solvente apolar, e no caso da extração em Soxhlet, a sua eficiência no método está relacionada ao seu maior tempo de operação e de volume de solvente assim como sua recirculação no processo, quando comparado com a extração supercrítica.

A Tabela 3 também apresenta os resultados de TFT dos extratos obtidos por diferentes condições operacionais da ESC. De acordo com a Tabela 3, o maior valor obtido do TFT na extração supercrítica foi na condição de 300 bar e $60{ }^{\circ} \mathrm{C}(23 \pm 1 \mathrm{mg}$ EAG/g). 
Tabela 3 - Teor de compostos fenólicos totais (TFT) nas extrações convencionais

\begin{tabular}{|c|c|c|c|}
\hline \multicolumn{3}{|c|}{ Condições de Extração } & \multirow[b]{2}{*}{ TFT (mg EAG/g) ${ }^{(1)}$} \\
\hline Técnica & $\begin{array}{l}\text { Secagem da } \\
\text { matéria-prima }\end{array}$ & $\begin{array}{l}\text { Solvente/Condições de } \\
\text { pressão e temperatura }\end{array}$ & \\
\hline \multirow{6}{*}{ Soxhlet } & \multirow{3}{*}{ Natural } & Etanol/atm/ebulição & $25^{\mathrm{c}} \pm 2$ \\
\hline & & Hexano/atm/ebulição & $2,3^{\mathrm{f}} \pm 0,3$ \\
\hline & & Ac. Etila/atm/ebulição & $21^{\mathrm{cd}} \pm 3$ \\
\hline & \multirow{3}{*}{ Estufa } & Etanol/atm/ebulição & $10,3^{\mathrm{de}} \pm 1,4$ \\
\hline & & Hexano/atm/ebulição & $18,3^{\mathrm{d}} \pm 1,5$ \\
\hline & & Ac. Etila/atm/ebulição & $30^{\mathrm{c}} \pm 1$ \\
\hline \multirow{6}{*}{ Maceração } & \multirow{3}{*}{ Natural } & Etanol/atm/ebulição & $63^{\mathrm{a}} \pm 2$ \\
\hline & & Hexano/atm/ebulição & $1,2^{\mathrm{f}} \pm 0,3$ \\
\hline & & Ac. Etila/atm/ebulição & $43^{b} \pm 2$ \\
\hline & \multirow{3}{*}{ Estufa } & Etanol/atm/ebulição & $45,2^{b} \pm 3,5$ \\
\hline & & Hexano/atm/ebulição & $5,6^{\mathrm{e}} \pm 0,3$ \\
\hline & & Ac. Etila/atm/ebulição & $54^{\mathrm{b}} \pm 3$ \\
\hline \multirow{6}{*}{ Ultrassom } & \multirow{3}{*}{ Natural } & Etanol/atm/ebulição & $20^{\mathrm{d}} \pm 2$ \\
\hline & & Hexano/atm/ebulição & $8,4^{\mathrm{e}} \pm 1,1$ \\
\hline & & Ac. Etila/atm/ebulição & $10,5^{\mathrm{e}} \pm 1,2$ \\
\hline & \multirow{3}{*}{ Estufa } & Etanol/atm/ebulição & $62,4^{\mathrm{a}} \pm 3,5$ \\
\hline & & Hexano/atm/ebulição & $6,4^{\mathrm{e}} \pm 2,1$ \\
\hline & & Ac. Etila/atm/ebulição & $5^{\mathrm{e}} \pm 1$ \\
\hline \multirow{5}{*}{ Extração Supercrítica } & \multirow{5}{*}{ Estufa } & $\mathrm{CO}_{2} / 100 \mathrm{bar} / 40^{\circ} \mathrm{C}$ & $3^{\mathrm{d}} \pm 2$ \\
\hline & & $\mathrm{CO}_{2} / 200 \mathrm{bar} / 50{ }^{\circ} \mathrm{C}$ & $7^{\mathrm{bc}} \pm 4$ \\
\hline & & $\mathrm{CO}_{2} / 300 \mathrm{bar} / 40{ }^{\circ} \mathrm{C}$ & $1,3^{\mathrm{dc}} \pm 0,3$ \\
\hline & & $\mathrm{CO}_{2} / 300 \mathrm{bar} / 50{ }^{\circ} \mathrm{C}$ & $15,8^{\mathrm{ab}} \pm 0,5$ \\
\hline & & $\mathrm{CO}_{2} / 300 \mathrm{bar} / 60{ }^{\circ} \mathrm{C}$ & $23^{\mathrm{a}} \pm 1$ \\
\hline
\end{tabular}

(1) Letras iguais na mesma coluna indicam que não há diferença significativa $(\mathrm{p}<0,05)$.

Em relação ao teor de fenólicos totais, o extrato obtido nos dois tipos de secagem da casca de manga e em todos os métodos de extração a baixa pressão, o uso do etanol como solvente se mostrou como melhor solvente para a concentração destes compostos, com destaque para as extrações Soxhlet e ultrassom das cascas secas por estufa. Avaliando o resultado geral do TFT, verifica-se que a presença de compostos fenólicos é observada em maior valor nos solventes de alta e média polaridade, não sendo, portanto, facilmente extraíveis por solventes de baixa polaridade ou apolares.

\section{CONCLUSÃO}

O resíduo agroindustrial (casca) da manga Tommy Atkins (Mangifera indica Linn) se apresentou como uma matéria prima viável para a obtenção de compostos fenólicos nos diferentes 


\section{9 a 22 de outubro de 2014 \\ Florianópolis/SC}

métodos de extração empregados. O sistema Soxhlet apresentou os melhores rendimentos de extração de casca de manga, especialmente quando o solvente empregado foi o etanol. Já na extração supercrítica foram obtidos rendimentos comparáveis a extração com hexano, sendo o maior valor obtido na condição de 300 bar $/ 50{ }^{\circ} \mathrm{C}(3,8 \pm 0,7 \%)$. O uso de etanol como solvente (em todas as técnicas a baixa pressão empregadas) se mostrou como melhor para concentrar compostos fenólicos da casca de manda, independente do tipo de secagem empregado. Na extração supercrítica o maior valor obtido do TFT foi a 300 bar e $60{ }^{\circ} \mathrm{C}(23 \pm 1 \mathrm{mg}$ EAG/g).

De acordo com os resultados mostrando observa-se que a casca de manga pode ser considerada uma matéria prima interessante para a obtenção de extratos de alto valor agregado (como compostos fenólicos) e viável economicamente no ponto de vista do aproveitamento de um descarte agroindustrial. Além disso, é observado que a matéria-prima tem afinidade por solvente de maior polaridade, pois tanto o Rendimento como o TFT tiveram melhores resultados com os solventes de maior polaridade. O emprego da ESC como tecnologia limpa para obtenção de extratos de alto valor agregado é indicado por ser um método ambientalmente seguro, pois não libera resíduo tóxico no ambiente e não contamina o extrato.

\section{REFERÊNCIAS}

AOAC. Association of Official Analytical Chemists - Official Methods of Analysis of the AOAC International. 18. ed. Maryland: AOAC, 2005.

ANGUS, S.; ARMSTRONG, B.; DE REUCK, K. M. International thermodynamic tables of the fluid state: Carbon dioxide. Oxford: Pergamon Press, 1976.

BYERS, J. A. Phenomex catalogue. Disponível em: <http://www. phenomenex.com/phen/Doc/z366.pdf.>. Acesso em: Julho. 2013.

BE-JEN, W.; YEN-HUI, L.; ZER-RAN, Y. Supercritical fluid extractive fractionation: study of the antioxidant activities of propolis. Food Chem., v.86 (2), p. 237-43, 2004.

COELHO, E.M., AZEVEDO,L.C. Comparação entre técnicas de secagem para obtenção de farinha a partir da casca de manga cv. Tommy Atkins; VII CONNEPI, 2012.

FONSECA, N, NETO,M.T. de C., LEDO,C.A.da S. Paclobutrazol e estresse hídrico no florescimento e produção da mangueira (mangifera indica) 'tommy atkins' Rev. Bras. Frutic., v. 27, n. 1, p. 21-24, 2005.

GU, Z.; CHEN, D.; HAN, Y.; CHEN, Z;. GU, F. Optimization of carotenoids extraction from Rhodobacter sphaeroides. LWT. v. 41, p. 1082-1088, 2008.

KHAJEH, M., YAMINI, Y., SEFIDKON, F., BAHRAMIFAR, N. Comparision of essencial oil composition of Carum copticum obtained by supercritical carbon dioxide extraction and hydrodistillation methods. Food Chem. v. 86, p 587-591, 2004.

KIM, H.; KIM, H.;MOSADDIK, A.; GYAWALI, R.,; AHN,K. S.; CHO, S.K. ; Induction of apoptosis by ethanolic extract of mango peel and comparative analysis of the chemical constitutes of mango peel and flesh. Food Chemistry,2012. 
PESCHEL, W.; SÁNCHEZ-RABANEDA, F.; DIEKMANN, W.; PLESCHER, A.; GARTZÍA, I.; JIMÉNEZ, D.; LAMUELA-RAVENTÓS, R.; BUXADERAS, S.; CODINA, C. An industrial approach in the search of natural antioxidants from vegetable and fruit wastes. Food Chem., v. 97, p. 137-50, 2006.

POURMORTAZAVI, S. M.; HAJIMIRSADEGUI, S. H. Supercritical fluid extraction in plant essential and volatile oil analysis. J. Chromatogr. A, v.1163, p. 2-24, 2007.

ROSSI, J. A. J.; SINGLETON, V. L. Colorimetry of total phenolics with phosphomolybdic phosphotungstic acid reagents. American Journal Enol. Vitic. v. 16, p. 144-58, 1965.

SACHINDRA, N.M.; BHASKAR, N.; MAHENDRAKAR, N.S. Recovery of carotenoids from shrimp waste in organic solvents. Waste Management, v. 26, p. 1092-1098, 2006. 